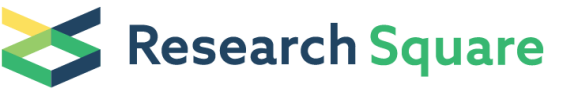 \\ Preprints are preliminary reports that have not undergone peer review. \\ They should not be considered conclusive, used to inform clinical practice, or referenced by the media as validated information.
}

\section{Hybrid Imaging Reveals Improved Absorbed Dose from Selective Internal Radiation Treatment by Using an Anti- Reflux Catheter}

\section{Esmaeel Jafargholi Rangraz}

KU Leuven: Katholieke Universiteit Leuven

\section{Xikai Tang}

KU Leuven: Katholieke Universiteit Leuven

\section{Geert Maleux}

UZ Leuven: Katholieke Universiteit Leuven Universitaire Ziekenhuizen Leuven

\section{Jeroen Dekervel}

UZ Leuven: Katholieke Universiteit Leuven Universitaire Ziekenhuizen Leuven

\section{Eric Van Cutsem}

UZ Leuven: Katholieke Universiteit Leuven Universitaire Ziekenhuizen Leuven

\section{Chris Verslype}

UZ Leuven: Katholieke Universiteit Leuven Universitaire Ziekenhuizen Leuven

\section{Kristof Baete}

UZ Leuven: Katholieke Universiteit Leuven Universitaire Ziekenhuizen Leuven

\section{Johan Nuyts}

KU Leuven: Katholieke Universiteit Leuven

\section{Christophe M Deroose ( $\nabla$ christophe.deroose@uzleuven.be)}

Katholieke Universiteit Leuven Universitaire Ziekenhuizen Leuven https://orcid.org/0000-0002-6080-1577

\section{Case report}

Keywords: hybrid imaging, radioembolization, selective internal radiation therapy (SIRT), liver perfusion territory, conebeam CT, dose volume histogram (DVH), predictive dosimetry, post-treatment dosimetry, voxel-level dosimetry, antireflux catheter

Posted Date: December 8th, 2020

DOI: https://doi.org/10.21203/rs.3.rs-122978/v1

License: (c) (i) This work is licensed under a Creative Commons Attribution 4.0 International License. Read Full License 


\section{Abstract}

Selective internal radiation therapy (SIRT) is a promising technique for patients with hepatic malignancies. Several image-based investigations, e.g. volumetric and absorbed dose assessment, are mandatory for SIRT planning and treatment verification based on national and international regulations.

General treatment workflows are described in guidelines, recommendations, and the package inserts of the manufactures. But, guidance to tackle particular clinical conditions can be ill-defined and different centers practice their own workflow to analyze the treatment process. This case report includes an example of inconsistency between treatment simulation and observed treatment result, revealed by hybrid imaging. There is no universally accepted standard procedure defined in the literature for detecting and evaluating a possible mismatch between [ $\left.{ }^{99 \mathrm{~m}} \mathrm{Tc}\right] \mathrm{Tc}-$ MAA-based simulation and distribution of the therapeutic microspheres. In this setting, more advanced multi-modal image-based analysis may be beneficial.

A 78 year old patient with hepatocellular carcinoma underwent liver radioembolization with resin ${ }^{90} \mathrm{Y}$-microspheres. Tumoral and non-tumoral dose-volume histograms were evaluated for simulated activity distribution using

$\left[{ }^{99 m}\right.$ Tc]TC-MAA-SPECT and post-treatment activity measurement using ${ }^{90} \mathrm{Y}$-PET. During simulation workup, [ $\left.{ }^{99 \mathrm{~m}} \mathrm{Tc}\right] \mathrm{Tc}-$ MAA particles were administered using a regular catheter. On the other hand, for the treatment session an anti-reflux catheter was used. Our result, suggests that the use of an anti-reflux catheter might improve tumor coverage, and as a result decrease non-tumoral liver uptake deposition.

\section{Introduction}

A workflow for selective internal radiation therapy (SIRT) using resin ${ }^{90}$ Y-microspheres (SIR Spheres, SIRTEX, Sydney, Australia) is described in the package insert and training program of the manufacturer [1], guidelines and recommendations by different associations [2, 3]. In SIRT, the absorbed dose (i.e. the deposited energy per unit of mass) is used to quantify the irradiation of the tumor and non-tumoral healthy liver [4].

In the daily clinical practice, each patient has unique clinical characteristics and needs, necessitating a dosimetrybased tailored activity planning strategy, and post-treatment dose verification [4]. This requires (1) a careful treatment planning using a simulation workup to estimate the intra- and extrahepatic distribution of the therapeutic microspheres, and (2) a post-treatment determination of the radionuclide distribution to identify potential adverse effects (treatment efficacy and safety).

In our previous studies, a personalized multi-modal dosimetry workflow was introduced [5, 6], and some patient population studies on comparing voxel-level pre-treatment predictive and post-treatment measured absorbed dose were presented [7]. Our proposed workflow uses automated segmentation and non-rigid image registration. In daily clinical routine, we apply this workflow in parallel to the standard clinical workflow, which relies on manual segmentation and visual inspection of all images.

Here, we describe a case to illustrate the usefulness of multi-modal image analysis, e.g. volume of interest (VOI) definition based on cone-beam CT (CBCT), in comparing predicted and measured dose. This case illustrates the value of using an anti-reflux catheter in SIRT for better tumor coverage and resulting efficacy/toxicity balance [8].

\section{Case Description}


A SIRT session was performed on a 78 year old patient with hepatocellulcar carcinoma (HCC). Based on diagnostic MRI and CT images, a large central (diameter: $8.6 \mathrm{~cm}$ ) and two satellite tumors, involving segments $/ /$ and $I V$, were diagnosed. The case was subsequently discussed at the multi-disciplinary tumor board. The tumor load was considered too extensive for transarterial chemoembolization (TACE). Hence, SIRT was considered as an alternative aiming at controlling tumor progression.

The treatment was planned based on a pre-SIRT workup using technetium-99m macro-aggregated albumin $\left(\left[{ }^{99 m}\right.\right.$ Tc]Tc-MAA) as a surrogate for therapeutic microspheres. During the workup, a super-selective catheterization of the hepatic artery (HA) was performed, and the perfusion territories of the left and right HA were evaluated. Dualphase CBCTs indicated the bulky tumor in segment $I V$ and two other smaller tumors. The tumors were irrigated from both the left and right HA. An accessory branch to segment / did not contribute to the blood flow of the tumors. Afterwards, a [99m Tc]Tc-MAA-SPECT/CT was performed, which predicted poor targeting, particularly within the right liver perfusion territory (LPT). In this LPT, almost no difference was found between the ventrally located tumor and dorsal healthy liver. An estimated lung shunt fraction (eLSF) of $4.8 \%$ was calculated.

The treatment was planned a month after [ $\left.{ }^{99 \mathrm{~m}} \mathrm{Tc}\right] \mathrm{Tc}-\mathrm{MAA}$ workup by using the Medical Internal Radiation Dose (MIRD) formalism with an entire liver absorbed dose of $50 \mathrm{~Gy}$ (equivalent to $1 \mathrm{MBq} / \mathrm{ml}$ infused volume). The therapeutic microspheres were administered in a comparable manner to the workup, except for one difference; an anti-reflux catheter (Surefire Infusion System; Surefire Medical, Westminster, Colorado) was used during the treatment session. This was done because a very narrow right gastric artery did branch from the left hepatic artery just after its origin; this vessel was too narrow to insert a catheter and occlude it with coils.

We hypothesized that the microsphere distribution with the use of an anti-reflux catheter could be different, in an unpredictable manner, compared to a regular micro-catheter. Interestingly, on the day of the treatment, preferential tumor targeting was observed. We performed detailed dosimetry, particularly with respect to predictive versus measured dose comparison.

Three months after treatment, an arterial and venous phase contrast-enhanced CT imaging and contrast- enhanced MR images were performed. The two satellite lesions showed a marked partial response (according to modified RECIST; mRECIST). For the big tumor, no volume increase was observed, and it was as also a partial response (mRECIST) due to its increasing central necrosis (see Fig. 1).

\section{Dosimetry Report}

By using a projected absorbed dose of $50 \mathrm{~Gy}$ (or $1 \mathrm{MBq}$ per $\mathrm{ml}$ ) to the entire liver, including tumor volume (TV) and non-tumor volume (NTV), 0.522 and $1.027 \mathrm{GBq}$ of therapeutic microspheres were prescribed for the left and right liver LPT, respectively.

Fractional uptakes were derived from the $\left[{ }^{99 \mathrm{~m}} \mathrm{Tc}\right] \mathrm{Tc}-\mathrm{MAA}-\mathrm{SPECT}$ and ${ }^{90} \mathrm{Y}$-PET for the predictive and post-treatment dosimetry, respectively. We performed dosimetry based on the local deposition model by utilizing these VOIs [7]:

- liver: the liver was delineated by applying a convolutional neural network on the CT image from the [ $\left.{ }^{99 m} \mathrm{Tc}\right] \mathrm{Tc}$ MAA workup with some manual adjustment [6],

- tumor: tumors were segmented on cone-beam CTs (CBCTs),

- LPTs: left and right LPTs were segmented on the CBCT images. 
For the post-treatment dosimetry, all the VOIs were aligned to the post-treatment 90Y-PET using non- rigid registration $[5,7]$. A comparison between segmented volumes used in the pre- and post-treatment dosimetry, together with the manual segmentation (which was used for prescription) are provided in Table 1; screenshots of three different transverse slices of various images, as well as defined VOls are shown in Fig. 2.

Table 1

Segmentation analysis; comparing our segmentation results and clinical records.

\begin{tabular}{|c|c|c|c|c|}
\hline parameter & method & left LPT & right LPT & entire liver \\
\hline \multirow[t]{3}{*}{$\mathrm{TV}(\mathrm{cc})$} & clinical routine & - & - & - \\
\hline & predictive dosimetry & 166 & 202 & 368 \\
\hline & post-treatment dosimetry & 162 & 200 & 366 \\
\hline \multirow[t]{3}{*}{ NTV (cc) } & clinical routine & - & - & - \\
\hline & predictive dosimetry & 318 & 822 & 1140 \\
\hline & post-treatment dosimetry & 345 & 856 & 1202 \\
\hline \multirow[t]{3}{*}{ entire VOI volume (cc) } & clinical routine & 520 & 1000 & 1520 \\
\hline & predictive dosimetry & 484 & 1024 & 1508 \\
\hline & post-treatment dosimetry & 507 & 1057 & 1564 \\
\hline \multirow[t]{3}{*}{ tumor involvement (\%) } & clinical routine & - & - & - \\
\hline & predictive dosimetry & 34 & 20 & 24 \\
\hline & post-treatment dosimetry & 32 & 19 & 23 \\
\hline \multirow[t]{3}{*}{ left to entire volume ratio (\%) } & clinical routine & 34 & - & - \\
\hline & predictive dosimetry & 32 & - & - \\
\hline & post-treatment dosimetry & 32 & - & - \\
\hline
\end{tabular}

A comparison between the predictive dosimetry and measured dose distribution is provided in Table 2. Cumulative dose-volume histogram are also provided in Fig. 3. The voxel-level dosimetry revealed that:

- TV mean dose in the post-treatment is notably higher than the pre-treatment evaluation for the right LPT (72 versus $189 \mathrm{~Gy}$ ). In the left LPT, the underestimation of the TV dose by [ ${ }^{99 \mathrm{~m}} \mathrm{Tc}$ ]Tc-MAA was less notable (82 versus $108 \mathrm{~Gy})$.

- more than $56 \%$ and $83 \%$ of the tumor volume received a dose of 100 Gy or more in the left and right LPTs, resulting in $70 \%$ for the entire liver.

- $85 \%$ of the NTV in the entire liver received an absorbed dose of less than $30 \mathrm{~Gy}$ (with a mean dose of only $17 \mathrm{~Gy}$ ). 


\begin{tabular}{|c|c|c|c|c|c|c|c|c|c|}
\hline \multirow[t]{2}{*}{ criterion } & \multicolumn{3}{|l|}{ left LPT } & \multicolumn{2}{|c|}{ right LPT } & \multicolumn{4}{|c|}{ entire liver } \\
\hline & predictive & $\begin{array}{l}\text { Post } \\
\text { treatment }\end{array}$ & $\Delta$ & predictive & $\begin{array}{l}\text { Post } \\
\text { treatment }\end{array}$ & $\Delta$ & predictive & $\begin{array}{l}\text { Post } \\
\text { treatment }\end{array}$ & $\Delta$ \\
\hline $\begin{array}{l}\text { TV } \\
\text { activity } \\
\text { (MBq/Cc) }\end{array}$ & 1.70 & 2.25 & 0.55 & 1.50 & 3.94 & 2.44 & 1.59 & 3.14 & 1.55 \\
\hline $\begin{array}{l}\text { NTV } \\
\text { activity } \\
\text { (MBq/CC) }\end{array}$ & 0.75 & 0.46 & -0.29 & 0.91 & 0.31 & -0.60 & 0.87 & 0.35 & -0.52 \\
\hline $\begin{array}{l}\text { T/N } \\
\text { ratio* } \\
\text { (ratio) }\end{array}$ & 2.26 & 4.91 & 2.65 & 1.65 & 12.88 & 11.23 & 1.84 & 9.00 & 7.16 \\
\hline $\begin{array}{l}\text { VOI dose } \\
\text { (Gy) }\end{array}$ & 52 & 49 & -3 & 49 & 48 & -1 & 50 & 48 & -2 \\
\hline $\begin{array}{l}\text { NTV } \\
\text { dose } \\
\text { (Gy) }\end{array}$ & 36 & 22 & -14 & 44 & 15 & -29 & 42 & 17 & -25 \\
\hline $\begin{array}{l}\text { TV dose } \\
\text { (Gy) }\end{array}$ & 82 & 108 & 26 & 72 & 189 & 117 & 76 & 153 & 77 \\
\hline $\begin{array}{l}\mathrm{V}^{3} 0_{\mathrm{NTV}}{ }^{\star *} \\
(\%)\end{array}$ & 42 & 23 & -19 & 74 & 11 & -63 & 65 & 15 & -50 \\
\hline $\begin{array}{l}\mathrm{V} 100_{\mathrm{TV}}{ }^{* \star} \\
(\%)\end{array}$ & 23 & 56 & 33 & 14 & 83 & 69 & 18 & 70 & 52 \\
\hline \multicolumn{10}{|c|}{ * $\mathrm{T} / \mathrm{N}$ ratio: tumor to normal tissue activity concentration ratio } \\
\hline
\end{tabular}

Table 2 Overview of the predictive and measured dosimetry comparison.

\section{Discussion}

In SIRT, the pre-treatment workup is the basis for the treatment planning. This investigation consists of two essential imaging studies: (a) an angiography to assess the gross hepatic vascular anatomy and to investigate tumoral perfusion. In most centers nowadays, this angiography is followed by a dual-phase contrast-enhanced CBCT (early and late arterial phase), focusing on the perfusion territory of each catheter position. These images also indicate the hyper- or hypovascular nature of the tumor(s), (b) a planar imaging and SPECT/CT imaging after administration of surrogate particles ([99m Tc]Tc-MAA) at each proposed catheter position. We use these images to predict the intra- and extrahepatic distribution of the therapeutic microspheres.

A good predictive power of the [ $\left.{ }^{99 \mathrm{~m}} \mathrm{Tc}\right] \mathrm{Tc}-\mathrm{MAA}$ simulation in SIRT treatment planning was demonstrated in some studies. Strigari and Flamen reported a good correlation between [ $\left.{ }^{99 \mathrm{~m}} \mathrm{Tc}\right] \mathrm{Tc}-\mathrm{MAA}$ particles and therapeutic microsphere distribution for $80 \%$ and $100 \%$ of the cases, respectively $[8,9]$. Kao et al. confirmed this correspondence later for tumor absorbed dose [10, 11]. 
On the other hand, the inaccuracy in the prediction made using $\left[{ }^{99 \mathrm{~m}} \mathrm{Tc}\right] \mathrm{Tc}-\mathrm{MAA}$ has been reported in some studies. Possible reasons for a mismatch between [ ${ }^{99 m}$ Tc]Tc-MAA and therapeutic microspheres are: (i) the discrepancy between [ $\left.{ }^{99 \mathrm{~m}} \mathrm{Tc}\right] \mathrm{Tc}-\mathrm{MAA}$ and therapeutic microsphere in shape, size, and size distribution [12], the number of administered particles/microspheres [13], in vivo stability, and infusion rate, (ii) the administration close to an arterial bifurcation or a small arterial branch [12], (iii) temporary vessel spasm during the pre-treatment workup [14], and (iv) the difference in catheterization, such as the catheter tip positioning and the used catheter type [15].

In our previous study, we compared pre-treatment dose estimation and post-treatment dose measurement. In this study, VOIs were segmented using CBCT images, which was demonstrated to improve the accuracy of LPT delineation compared to anatomical lobe segmentation using contrast-enhanced CT images. We demonstrated a stronger agreement for NTV compartment and total LPT than for the TV.

Fortunately, ${ }^{90}$ Y-PET imaging revealed a more extensive tumor uptake with greater TV absorbed dose than that predicted by the workup (by a factor of 2.0), for the described patient. As a result, decreased activity deposition in the areas of NTV was measured compared to the pre-treatment workup. As the catheterization was similar for this patient, we believe that this large mismatch between [ $\left.{ }^{99 m} \mathrm{Tc}\right] \mathrm{Tc}-\mathrm{MAA}$ and therapeutic microsphere activity distributions was due to the use of anti-reflux catheter in the therapy session. Pasciak et al. also reported a similar effect with the use of an anti-reflux catheter, with an increase of tumor uptake with on average of 68\% (range 33$90 \%$ ) in 9 patients when comparing to an infusion with a conventional end-hole catheter [16].

\section{Conclusion}

Here, we present a case for which a multi-modal image processing facilitated the use of more information from all available images (e.g. CBCT, [ $\left.{ }^{99 m} \mathrm{Tc}\right] \mathrm{Tc}-\mathrm{MAA}-\mathrm{SPECT} / \mathrm{CT}$, and ${ }^{90} \mathrm{Y}-\mathrm{PET} / \mathrm{MR}$ ) to create more detailed reports. These details can help the physician to extract clinically relevant information, maximizing both therapy efficacy and safety.

\section{Acronyms}

${ }^{90} \mathrm{Y}$ yttrium-90.

[99m Tc]Tc-MAA technetium-99m macro-aggregated albumin.

CBCT cone-beam CT.

cDVH cumulative dose-volume histogram.

eLSF estimated lung shunt fraction.

HA hepatic artery.

HCC hepatocellular carcinoma.

LPT liver perfusion territory.

MIRD Medical Internal Radiation Dose.

NTV non-tumor volume. 
SIRT selective internal radiation therapy.

TACE transarterial chemoembolization.

TV tumor volume.

VOI volume of interest.

\section{Declarations}

Ethics approval and consent to participate

This study was approved by the Ethical committee research University Hospitals / KU Leuven.

Consent for publication

Not applicable.

Availability of data and material

The datasets of the images supporting the conclusions of this article may be made available upon request from the corresponding author.

\section{Competing interests}

CMD was a member of advisory boards for Novartis Pharma, Terumo, Ipsen and has been a consultant for Sirtex, Novartis Pharma and Bayer. All other authors do not have any conflicts of interest to disclose.

\section{Funding}

This project is supported by the Research Foundation- Flanders (FWO, grant G082418N) and by the European H2020 MSCA-ITN-2017 project (HYBRID, id 764458).

CMD is a Senior Clinical Investigator for the Research Foundation Flanders (FWO).

\section{Authors' contributions}

All authors contributed to the study design and manuscript editing and review. JN, CMD and KB are the principal investigators of the research and have been involved in revising the manuscript critically for important intellectual content. EJR, XT and JN did the software development. EJR was involved in data collection, image analysis, and drafting the manuscript. CMD performed VOI validation. KB designed the acquisition of [ ${ }^{99 m}$ Tc]Tc-MAA-SPECT/CT. GM performed the angiographical workups and angiographies during SIRT and performed the CBCTs. JDK, EVC, and CV performed patient selection, presentation at multidisciplinary tumor board, and patient care during pre-SIRT procedure and post-SIRT follow-up.

All authors read and approved the final manuscript.

\section{Acknowledgements}

The authors wish to thank Christelle Terwinghe, Wies Deckers (Nuclear Medicine, Department of imaging and pathology, UZ \& KU Leuven), and Janne Vignero (Radiology Section, Department of imaging and pathology, UZ 
Leuven, Leuven, Belgium) for the technical support.

\section{References}

Sirtex, SIR-Spheres microspheres (training program / Physicians and Instituions), Sirtex, 2015.

F. Giammarile, L. Bodei, C. Chiesa, G. Flux, F. Forrer, F. Kraeber-Bodere, B. Brans, B. Lambert, M. Konijnenberg, F. Borson-Chazot, J. Tennvall and M. Luster, "EANM Procedure Guideline for the Treatment of Liver Cancer and Liver Metastases with Intra-Arterial Radioactive Compounds," European Journal of Nuclear Medicine and Molecular Imaging, vol. 38, p. 1393-1406, 42011.

W. A. Dezarn, J. T. Cessna, L. A. DeWerd, W. Feng, V. L. Gates, J. Halama, A. S. Kennedy, S. Nag, M. Sarfaraz, V. Sehgal, R. Selwyn, M. G. Stabin, B. R. Thomadsen, L. E. Williams and R. Salem, "Recommendations of the American Association of Physicists in Medicine on dosimetry, imaging, and quality assurance procedures for ${ }^{90} \mathrm{Y}$ microsphere brachytherapy in the treatment of hepatic malignancies," Medical Physics, vol. 38, p. 4824-4845, 82011.

R. Bastiaannet, S. C. Kappadath, B. Kunnen, A. J. A. T. Braat, M. G. E. H. Lam and H. W. A. M. de Jong, "The Physics of Radioembolization," EJNMMI Physics, vol. 5, 112018.

E. J. Rangraz, W. Coudyzer, G. Maleux, K. Baete, C. M. Deroose and J. Nuyts, "Multi-modal image analysis for semiautomatic segmentation of the total liver and liver arterial perfusion territories for radioembolization," EJNMMI Research, vol. 9, 22019.

X. Tang, E. J. Rangraz, W. Coudyzer, J. Bertels, D. Robben, G. Schramm, W. Deckers, G. Maleux, K. Baete, C. Verslype, M. J. Gooding, C. M. Deroose and J. Nuyts, "Whole Liver Segmentation Based on Deep Learning and Manual Adjustment for Clinical Use in SIRT," European Journal of Nuclear Medicine and Molecular Imaging, vol. 46, pp. S763-S763, 42020.

E. J. Rangraz, X. Tang, C. V. Laeken, G. Maleux, J. Dekervel, E. V. Cutsem, C. Verslype, K. Baete, J. Nuyts and C. M. Deroose, "Quantitative comparison of pre-treatment predictive and post-treatment measured dosimetry for selective internal radiation therapy using cone-beam CT for tumor and liver perfusion territory definition," EJNMMI Research, vol. 10, 82020 .

L. Strigari, R. Sciuto, S. Rea, L. Carpanese, G. Pizzi, A. Soriani, G. laccarino, M. Benassi, G. M. Ettorre and C. L. Maini, "Efficacy and Toxicity Related to Treatment of Hepatocellular Carcinoma with ${ }^{90} \mathrm{Y}-\mathrm{SIR}$ Spheres: Radiobiologic Considerations," Journal of Nuclear Medicine, vol. 51, p. 1377-1385, 82010.

P. Flamen, B. Vanderlinden, P. Delatte, G. Ghanem, L. Ameye, M. V. D. Eynde and A. Hendlisz, "Multimodality Imaging Can Predict the Metabolic Response of Unresectable Colorectal Liver Metastases to Radioembolization Therapy with Yttrium-90 Labeled Resin Microspheres," Physics in Medicine and Biology, vol. 53, p. 6591-6603, 102008.

Y. H. Kao, "A Clinical Dosimetric Perspective Uncovers New Evidence and Offers New Insight in Favor of ${ }^{99 m}$ TcMacroaggregated Albumin for Predictive Dosimetry in ${ }^{90} \mathrm{Y}$ Resin Microsphere Radioembolization," Journal of Nuclear Medicine, vol. 54, p. 2191-2192, 112013.

Y.-H. Kao, J. D. Steinberg, Y.-S. Tay, G. K. Y. Lim, J. Yan, D. W. Townsend, C. A. Budgeon, J. A. Boucek, R. J. Francis, T. S. T. Cheo, M. C. Burgmans, F. G. Irani, R. H. G. Lo, K.-H. Tay, B.-S. Tan, P. K. H. Chow, S. Satchithanantham, A. E. H. Tan, 
D. C. E. Ng and A. S. W. Goh, "Post-Radioembolization Yttrium-90 PET/CT - Part 2: Dose-Response and Tumor Predictive Dosimetry for Resin Microspheres," EJNMMI Research, vol. 3, p. 57, 2013.

M. Wondergem, M. L. J. Smits, M. Elschot, H. W. A. M. de Jong, H. M. Verkooijen, M. A. A. J. van den Bosch, J. F. W. Nijsen and M. G. E. H. Lam, "99m Tc-Macroaggregated Albumin Poorly Predicts the Intrahepatic Distribution of ${ }^{90} \mathrm{Y}$ Resin Microspheres in Hepatic Radioembolization," Journal of Nuclear Medicine, vol. 54, p. 1294-1301, 62013.

C. Chiesa, M. Maccauro, R. Romito, C. Spreafico, S. Pellizzari, A. Negri, C. Sposito, C. Morosi, E. Civelli, R. Lanocita, T. Camerini, C. Bampo, S. Bhoori, E. Seregni, A. Marchianò, V. Mazzaferro and E. Bombardieri, "Need, feasibility and convenience of dosimetric treatment planning in liver selective internal radiation therapy with ${ }^{90} \mathrm{Y}$ microspheres: The experience of the National Cancer Institute of Milan," Quarterly Journal of Nuclear Medicine and Molecular Imaging, vol. 55, p. 168-197, 42011.

E. Garin, Y. Rolland, S. Laffont and J. Edeline, "Clinical Impact of ${ }^{99 m}$ Tc-MAA SPECT/CT-Based Dosimetry in the Radioembolization of Liver Malignancies with ${ }^{90}$ Y-Loaded Microspheres," European Journal of Nuclear Medicine and Molecular Imaging, vol. 43, p. 559-575, 92015.

M. Jiang, A. Fischman and F. S. Nowakowski, "Segmental Perfusion Differences on Paired Tc-99m Macroaggregated Albumin (MAA) Hepatic Perfusion Imaging and Yttrium-90 (Y-90) Bremsstrahlung Imaging Studies in SIR-Sphere Radioembolization: Associations with Angiography," Journal of Nuclear Medicine \& Radiation Therapy, vol. $03,2012$.

A. S. Pasciak, J. H. McElmurray, A. C. Bourgeois, R. E. Heidel and Y. C. Bradley, "The Impact of an Antireflux Catheter on Target Volume Particulate Distribution in Liver-Directed Embolotherapy: A Pilot Study," Journal of Vascular and Interventional Radiology, vol. 26, p. 660-669, 52015.

\section{Figures}

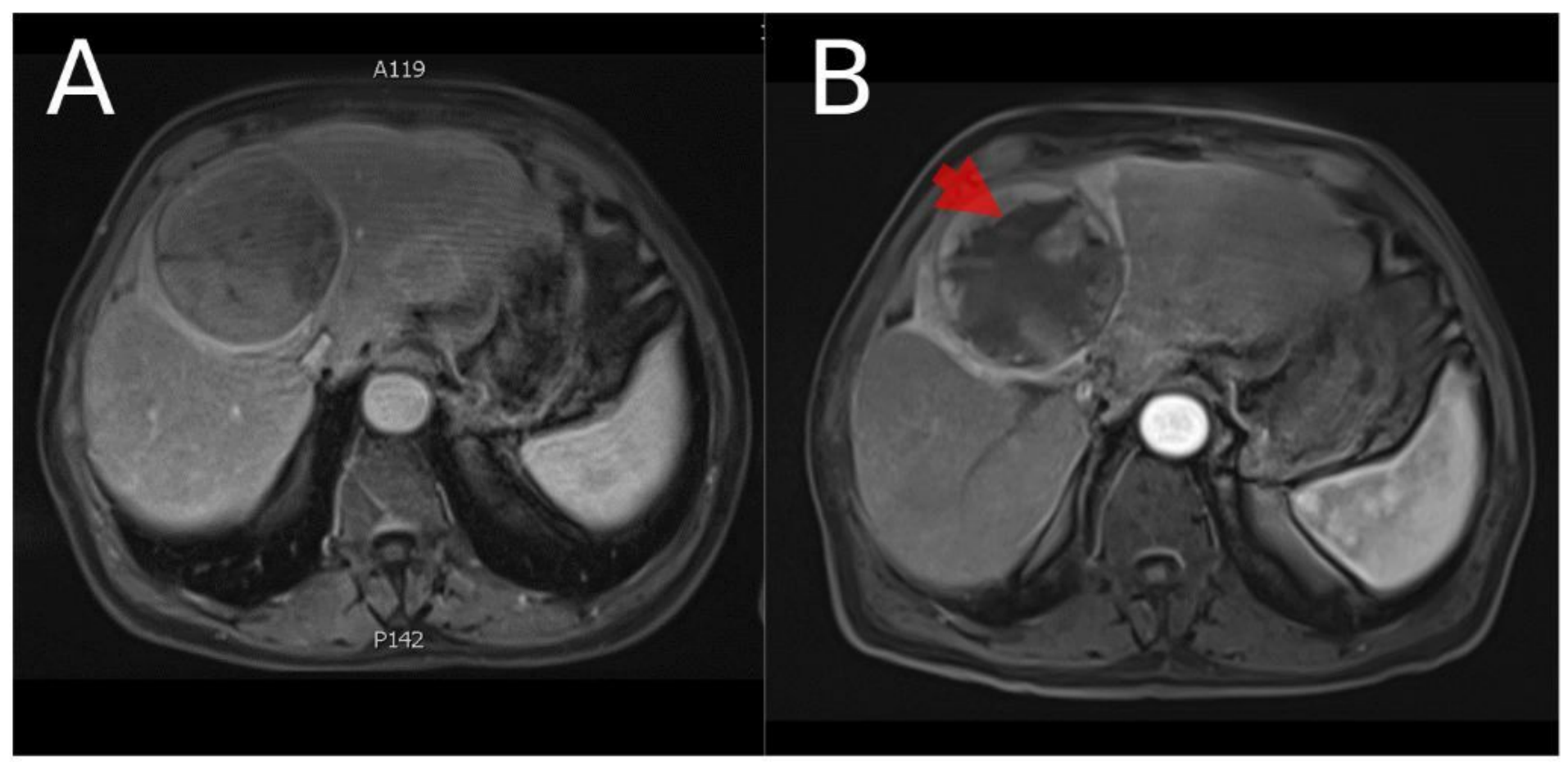

Figure 1 
[A] MR image before SIRT, performed 1.5 month before the treatment session, [B] a follow- up MR performed three months after the SIRT session. These images demonstrate the appearance of a prominent central necrosis (hypointense; see red arrow).

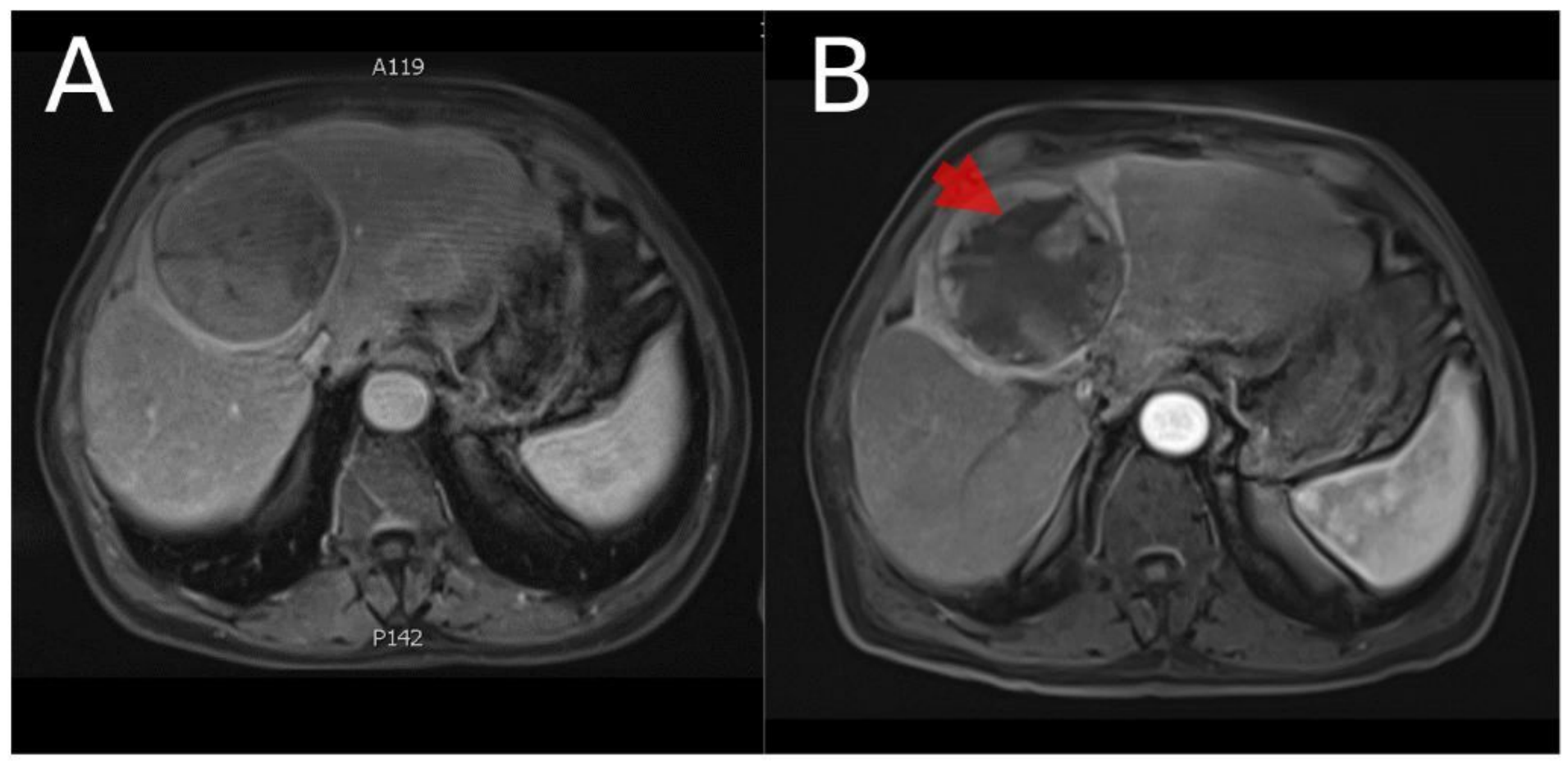

Figure 1

[A] MR image before SIRT, performed 1.5 month before the treatment session, [B] a follow- up MR performed three months after the SIRT session. These images demonstrate the appearance of a prominent central necrosis (hypointense; see red arrow). 


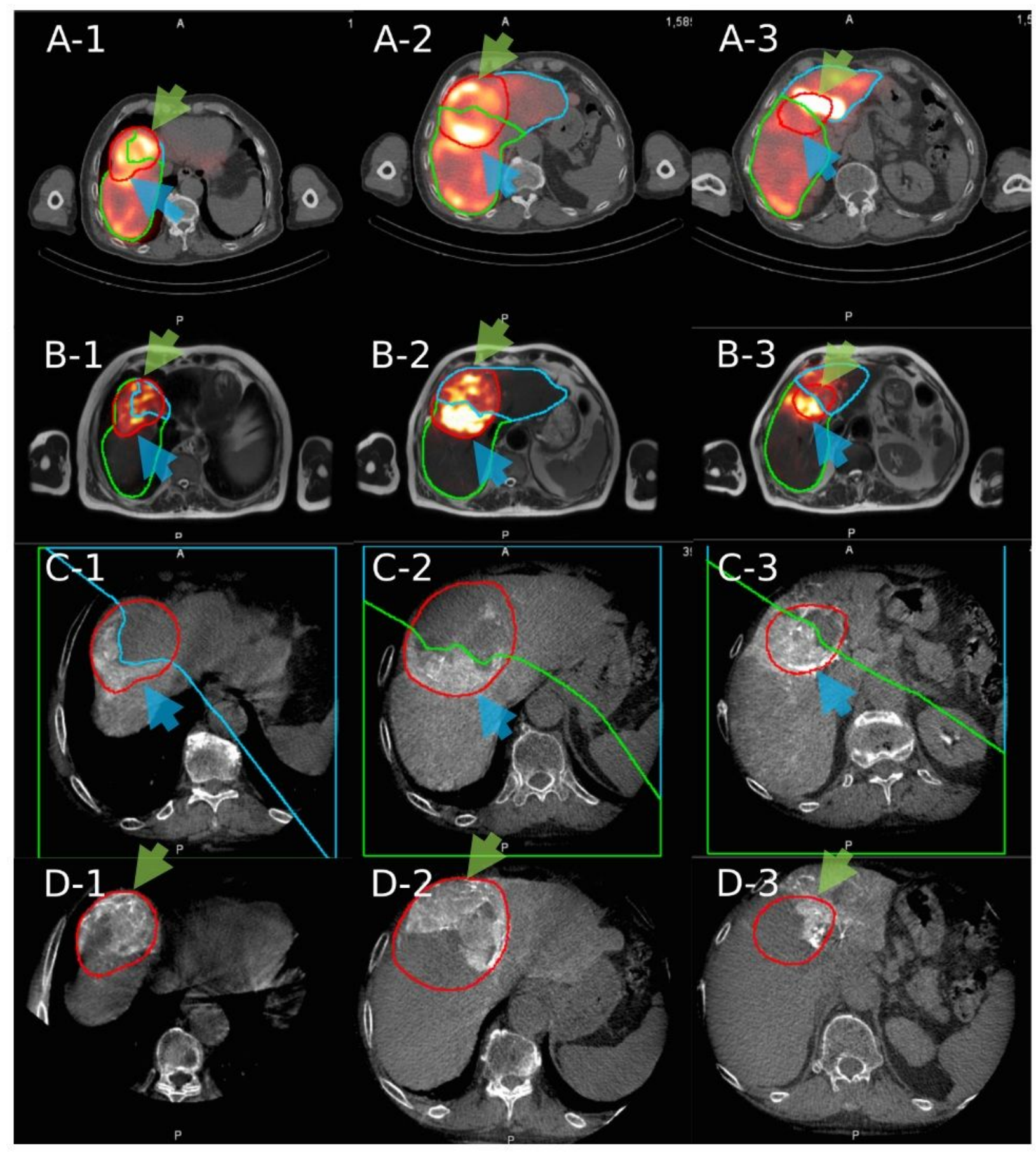

\section{Figure 2}

Three different slices of the images employed in dosimetry; the green, blue, and red contours outline the right LPT, left LPT, and tumor. [A-1 to A-3] the first row shows the [99mTc]Tc-MAA-SPECT/CT; [B-1 to B-3] the second row is the posttreatment 90Y-PET/MR; [C-1 to C-3] the third and [D-1 to D-3] fourth rows are late arterial phase CBCTs for the right and left perfusion territories. This figure represents the importance of the CBCT imaging not only in separating different perfusion territories but also in defining portions of the tumor that receive activity from various branches of the hepatic artery (green and blue arrow: volume of tumor perfused by the left and right hepatic artery). A visual comparison between the pre-treatment simulation ([99mTc]Tc-MAA-SPECT/CT) and post-treatment activity measurement (90Y-PET), indicates that the simulation underestimated the tumor uptake. 


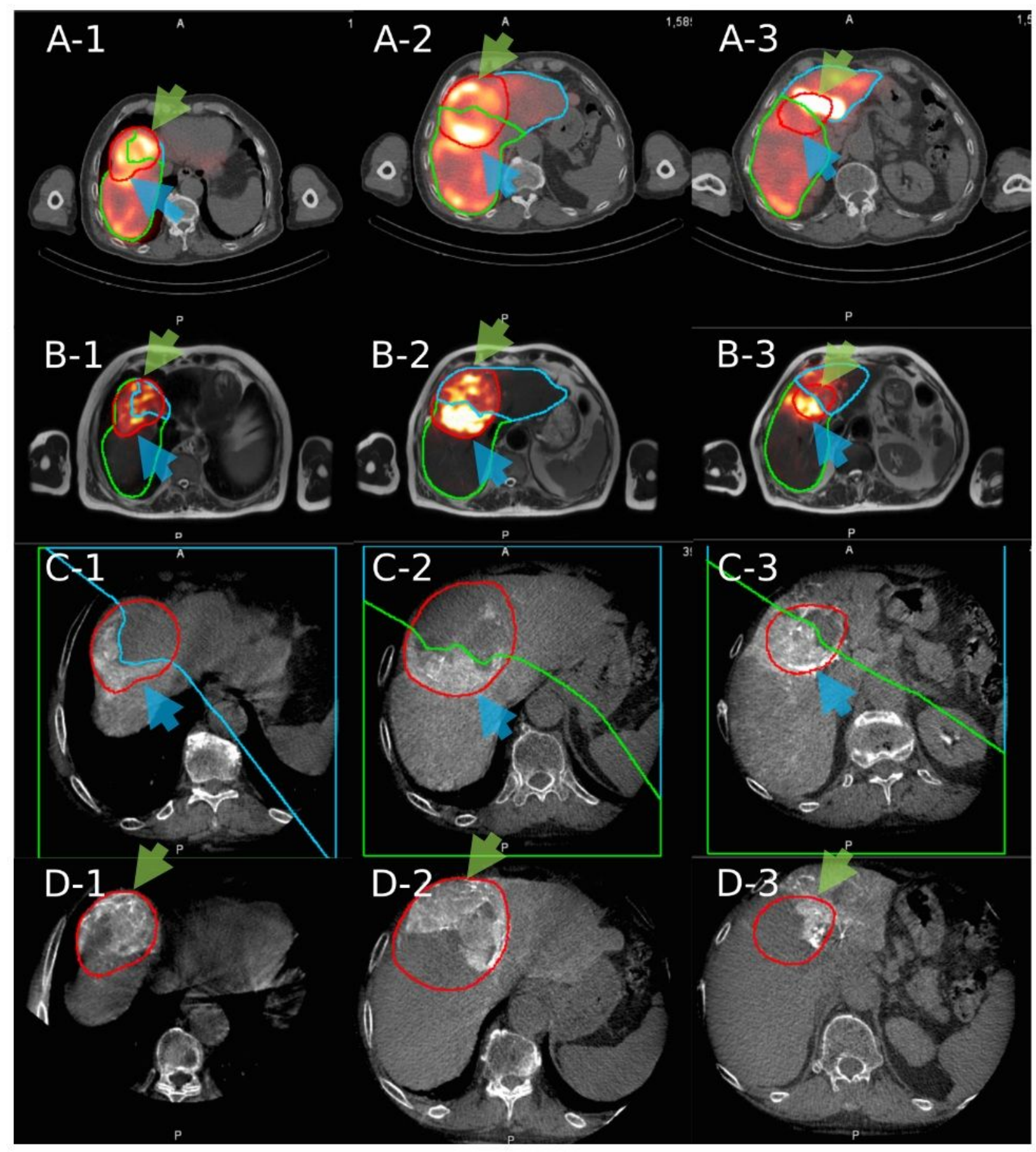

\section{Figure 2}

Three different slices of the images employed in dosimetry; the green, blue, and red contours outline the right LPT, left LPT, and tumor. [A-1 to A-3] the first row shows the [99mTc]Tc-MAA-SPECT/CT; [B-1 to B-3] the second row is the posttreatment 90Y-PET/MR; [C-1 to C-3] the third and [D-1 to D-3] fourth rows are late arterial phase CBCTs for the right and left perfusion territories. This figure represents the importance of the CBCT imaging not only in separating different perfusion territories but also in defining portions of the tumor that receive activity from various branches of the hepatic artery (green and blue arrow: volume of tumor perfused by the left and right hepatic artery). A visual comparison between the pre-treatment simulation ([99mTc]Tc-MAA-SPECT/CT) and post-treatment activity measurement (90Y-PET), indicates that the simulation underestimated the tumor uptake. 


\section{Predictive and measured cumulative DVH for different VOIs}

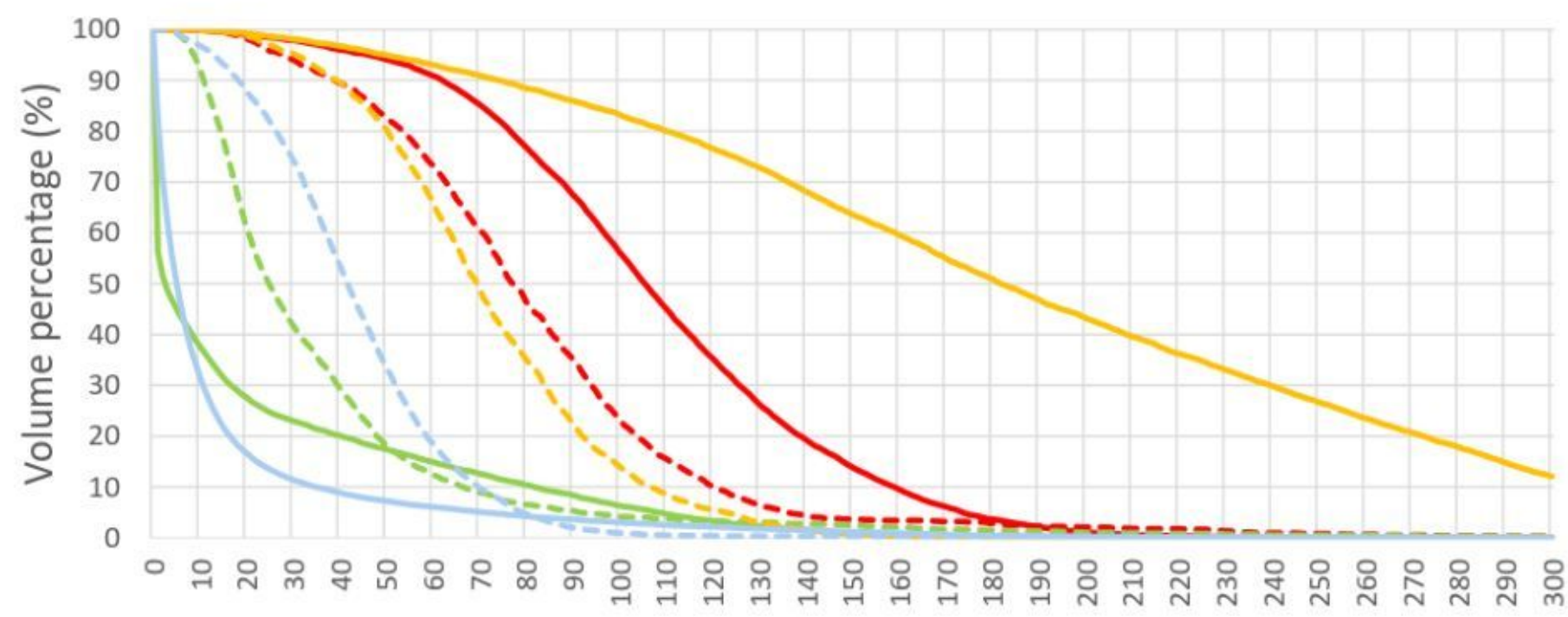

Absorbed dose (Gy)

- - left tumor (predictive)

- - left normal (predictive)

- - right tumor (predictive)

- - right normal (predictive)

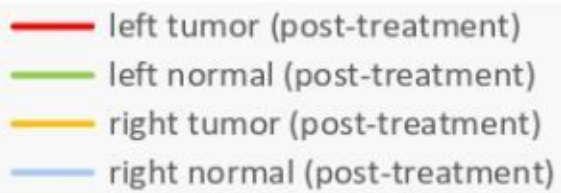

Figure 3

Cumulative dose-volume histogram showed that for both LPTs, predicted dosimetry underestimated the TV dose and overestimated the NTV dose significantly.

\section{Predictive and measured cumulative DVH for different VOIs}

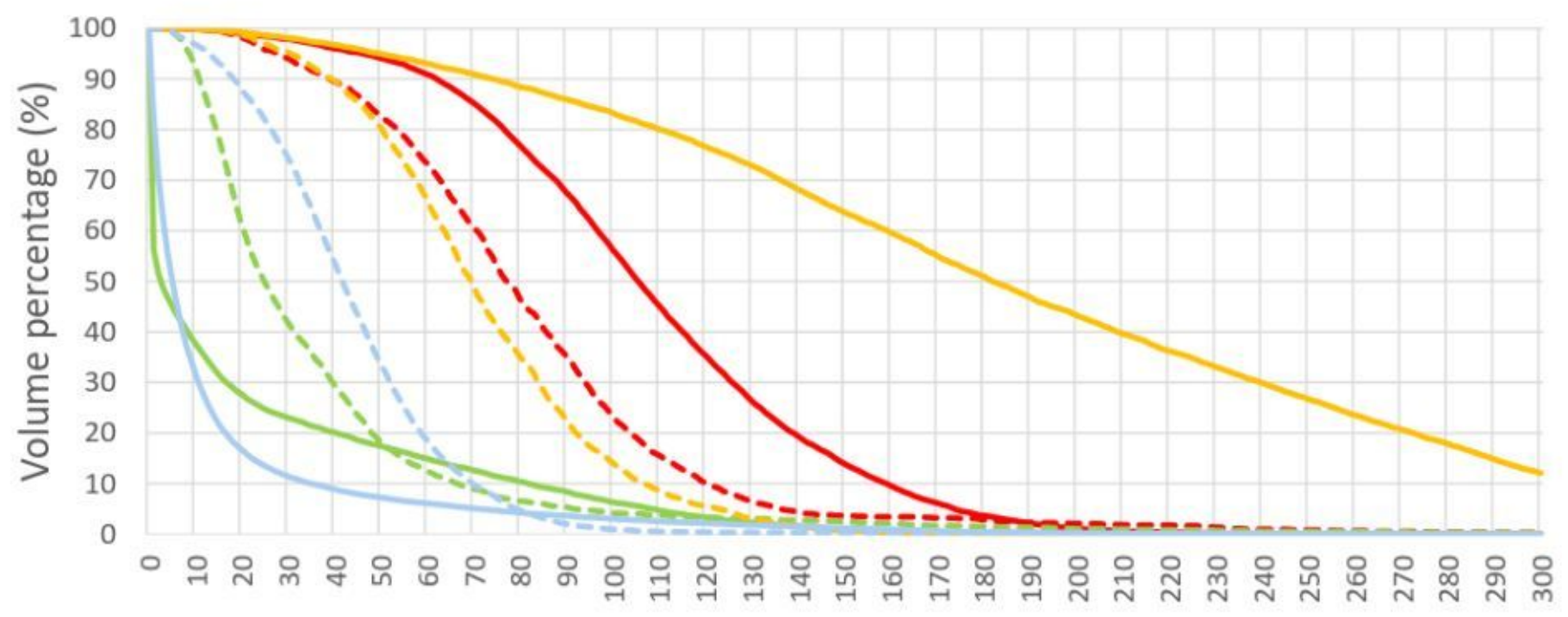

Absorbed dose (Gy)

- - left tumor (predictive)

- - left normal (predictive)

- - right tumor (predictive)

- - right normal (predictive)

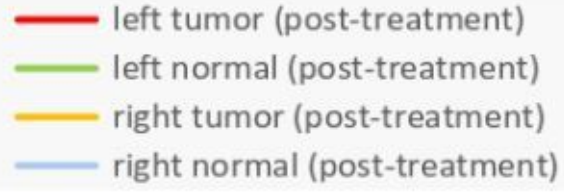




\section{Figure 3}

Cumulative dose-volume histogram showed that for both LPTs, predicted dosimetry underestimated the TV dose and overestimated the NTV dose significantly. 